\title{
Quantifying tracheobronchial tree dimensions: methods, limitations and
} emerging techniques

\author{
J.P. Williamson*,\# , A.L. James*, ${ }^{\uparrow,+}$, M.J. Phillips ${ }^{\S}$, D.D. Sampson ${ }^{\dagger}$, \\ D.R. Hillman*, and P.R. Eastwood ${ }^{*, \#, 9}$
}

ABSTRACT: The ability to measure airway dimensions is important for clinicians, interventional bronchoscopists and researchers in order to accurately quantify structural abnormalities and track their changes over time or in response to treatment.

Most quantitative airway measurements are based on X-ray computed tomography and, more recently, on multidetector computed tomography. Quantitative bronchoscopic techniques have also been developed, although these are less widely employed. Emerging techniques, including magnetic resonance imaging, endoscopic optical coherence tomography, endobronchial ultrasound and confocal endomicroscopy, provide new research tools with potential clinical applications.

An understanding of issues related to the acquisition, processing and analysis of images, and how such issues impact on imaging the tracheobronchial tree, is essential in order to assess measurement accuracy and to make effective use of the newer methods. This article contributes to this understanding by providing a comprehensive review of current and emerging techniques for quantifying airway dimensions.

KEYWORDS: Airway dimensions, confocal endomicroscopy, multidetector computed tomography, optical coherence tomography, videobronchoscopy

A dvances over the past 20 yrs in medical imaging and bronchoscopy have heralded an era of unprecedented access to quantitative information on tracheobronchial structure and, increasingly, function. The expanding scope of technical advances challenges the ability of clinicians and researchers to keep abreast of recent developments and emerging techniques. Computed tomography (CT) and, to a lesser extent, bronchoscopy are the two imaging tools that have contributed most to our understanding of the airway in vivo and are used on a daily basis by clinicians in the management of airway diseases. Newer techniques, such as magnetic resonance imaging, optical coherence tomography, fibreoptic confocal endomicroscopy and endobronchial ultrasound, offer fresh insights into the way we view and quantify the airway.
Over the coming years, the clinical applications of these newer techniques will be defined. As with endobronchial ultrasound, which has been adopted by interventional bronchoscopists in a little over a decade from its first reported use [1], it is likely that smoking-related lung diseases, such as bronchogenic cancer and chronic obstructive pulmonary disease (COPD), will drive the rapid development of new endoscopic imaging modalities and maintain the current pace of technical improvements of existing techniques, in particular CT. Initial applications of these techniques may focus on screening and surveillance of endobronchial malignancies and the use of real-time airway measurements during interventional procedures to deploy endobronchial stents. In these settings, accurate real-time quantitative imaging is advantageous.

\section{AFFILIATIONS}

Depts of *Pulmonary

Physiology, and

${ }^{\text {s}}$ Respiratory Medicine, Sir Charles

Gairdner Hospital,

'West Australian Sleep Disorders

Research Institute, Sir Charles

Gairdner Hospital

${ }^{\#}$ School of Anatomy \& Human

Biology, University of Western

Australia,

+School of Medicine and

Pharmacology, University of Western

Australia, and

${ }^{f}$ Optical+Biomedical Engineering

Laboratory, School of Electrical,

Electronic \& Computer Engineering, University of Western Australia, Perth, Australia.

\section{CORRESPONDENCE}

J.P. Williamson

Dept of Pulmonary Physiology

Sir Charles Gairdner Hospital

Nedlands

Perth 6009

Australia

E-mail: Jonathan.Williamson@

health.wa.gov.au

Received:

Feb 112008

Accepted after revision:

Jan 012009 
In this article, current and emerging methods of measurement of the tracheobronchial tree are critically appraised. Such methods should allow for accurate and reproducible airway measurements, and should be safe, clinician friendly and capture dynamic airway properties (table 1).

\section{GENERAL CONSIDERATIONS}

The tracheobronchial tree, also referred to in the present article as the lower airway, begins at the trachea, below the vocal cords, and extends to the terminal bronchioles in the lung periphery. Several systems of nomenclature may be used to describe this tree, depending on context. Bronchoscopists refer to the central versus peripheral airways, distinguished by what can be visually assessed during a procedure. From the trachea (generation 0), the airways divide into around 23 generations [2] and named airways down to the 6th generation have been described [3-5]. In the era of multidetector CT (MDCT) imaging, more airways can be imaged than are named, and a modified nomenclature system has been developed [6]. This system describes not only anatomical structures but also airway branch points to facilitate direct comparison of airway measurements within and between individuals over time and at varying lung volumes.

There are several dimensions of interest in an airway. In the large airways, diameter and cross-sectional area are most commonly measured and, in the setting of obstructing lesions, length and calibre of stenosed segments are important. As mentioned, branch points and branch length are of increasing interest. In smaller airways, in addition to measuring diameter and cross-sectional area, wall thickness is important, reflecting the changes of remodelling seen in asthma and COPD [7]. Smaller dimensions, such as basement membrane thickness, cannot, at present, be measured with standard imaging, although emerging technologies offer this capacity.

Imaging records structure and function in the context of procedure guidance or assessment of change in pathology over time or in response to treatment. Each of these imaging tasks can be classified as performing either detection or characterisation roles. Increasingly, both detection and characterisation incorporate computer algorithms which support the expert human observer. In assessing the utility of such computer support systems, their accuracy and precision must be

TABLE 1 Characteristics of an ideal quantitative airway
imaging tool
Readily repeatable measurements in airway sizes spanning the
tracheobronchial tree
Automated measurement of dimensions (e.g. lumen area and wall thickness)
Correction of angle of orientation, ensuring airways are measured in
cross section
Favourable inter-observer and intra-observer repeatability
Safe for long-term and repeated use (minimal levels of ionising radiation)
Measurements easily repeated before and after interventions
Dynamic acquisition so that functional, as well as structural, airway
information is available
Ability to segment the airway into a three-dimensional view
Provide detail of the subsurface architecture

evaluated. Accuracy is defined as the closeness of a measurement to the true value, whereas precision is the degree to which further measurements give the same result. Since absolute "true" measurements in biological systems are rarely possible (even the "gold standard" histology may be affected by sample shrinkage), measurements obtained using different imaging modalities may vary, their adequacy being determined by the research or clinical questions raised. Carefully constructed phantoms (models) are important to allow comparison between established and new imaging techniques [8]. The use of such calibration phantoms is particularly important to ensure standardisation of image-based acquisitions and analysis between devices from different companies and for multicentre studies, to reveal the relative strengths and limitations of each technique.

It is important to note that, although the tracheobronchial tree is often modelled as a branching structure composed of tapering round tubes, it is much more complex. At times airways may be ovoid in cross section (particularly at branch points) and any correction algorithm altering this cross section to appear more circular could be inappropriate. Furthermore, airway dimensions are not static but vary with regional anatomy (e.g. cartilaginous rings and external structures) as well as with lung volume and respiration. Therefore, for serial dimension comparisons between and within subjects, lung volume should be controlled. While spirometric gating is often used for this purpose $[9,10]$, the precise effect of lung volume on airway wall area is poorly understood. JAMES et al. [11] showed no change in airway wall area as lung volumes were reduced in guinea pigs but human studies are lacking and this remains an area of deficiency in the literature.

Quantitative imaging allows us to move beyond the traditional "global" assessment of lung properties to describe the "regional" characteristics, permitting better understanding of disease. Applications of quantitative airway measurements are diverse (table 2) and no imaging modality is ideal in all settings. Videobronchoscopy is useful for the assessment of airways with diameters down to $4-5 \mathrm{~mm}$ but, in routine clinical practice, bronchoscope-based measurements lack accuracy, and their precision is limited by operator subjectivity. MDCT, conversely, provides accurate measurements in these airways, although at the expense of ionising radiation and cannot readily be used during a procedure. Airways smaller than this are less well visualised with conventional bronchoscopes, although ultrafine paediatric bronchoscopes are able to assess more peripheral airways. In these smaller airways, highresolution CT (HRCT) and, more recently, MDCT can accurately provide structural detail in airways down to diameters of $2 \mathrm{~mm}$, and increasingly in airways smaller than this. Unlike videobronchoscopy, MDCT details relationships to adjacent structures and can measure wall thickness, branch points and branch length. Like videobronchoscopy, MDCT provides functional information by recording dynamic airway motion.

\section{QUANTITATIVE CT AIRWAY IMAGING}

CT provides a noninvasive method of quantifying airway dimensions and will be considered first. 
TABLE 2 Applications of quantitative airway measurements

\begin{tabular}{|c|c|c|}
\hline Application & Airway size & Current methods \\
\hline Identifying airway stenoses (e.g. malignancy, transplant & Large & MDCT \\
\hline anastamotic stricture) & & Virtual bronchoscopy \\
\hline \multirow[t]{2}{*}{ Airway stent selection (calibre and length) } & Large & MDCT aided by $3 \mathrm{D}$ rendering \\
\hline & & Videobronchoscopy \\
\hline Assessing endobronchial tumour size & & EBUS \\
\hline \multirow[t]{2}{*}{ Dynamic narrowing of bronchomalacia } & Large & Videobronchoscopy \\
\hline & Intermediate & MDCT \\
\hline Site of airway narrowing in obstructive lung diseases & Small, Intermediate & MDCT \\
\hline Measurement of airway narrowing during bronchoconstriction & Small, Intermediate & MDCT \\
\hline Measuring airway wall thickness in asthma/COPD & Small, Intermediate & MDCT \\
\hline
\end{tabular}

COPD: chronic obstructive pulmonary disease; MDCT: multidetector computed tomography; 3D: three dimensional; EBUS: endobronchial ultrasound.

\section{Principles of quantitative CT imaging}

The field of view from a CT cross-sectional (two-dimensional) image is usually displayed in a digital matrix of $512 \times 512$ pixels. Although CT images are generally viewed in a twodimensional format, they represent three-dimensional data, the third dimension being slice thickness. A voxel is the threedimensional equivalent of a pixel, combining the pixel area $(x$ and $y$ axis) with tomogram slice thickness ( $z$ axis). Consideration of the three-dimensional nature of CT is important when interpreting airway measurements, as will be discussed below. Collimation is a term often used interchangeably with slice thickness. It is more accurately defined as the width of the radiation detectors in the scanner. The scanner contains rows of radiation detectors (the detector array), which may vary in number from one (single detector) to 320 (multidetector). Greater detector numbers reduce image acquisition time and improve resolution. HRCT is a protocol developed using single-slice scanners to improve resolution. Individual axial slices of $1.0-2.0 \mathrm{~mm}$ thickness are scanned at $10-15 \mathrm{~mm}$ intervals and reconstructed using high spatial frequency algorithms to enhance edge detection. With the arrival of MDCT the HRCT protocol has largely been replaced. MDCT acquires contiguous $0.5-1.0-\mathrm{mm}$ lung slices in a single breath hold, generating a true volumetric data set that can be reconstructed in two dimensions through any plane. While MDCT represents the most powerful current technique for quantitatively assessing the airway in vivo, challenges exist and are discussed below.

\section{Limits of CT resolution}

Several factors influence the size of airways that can be resolved. During scanning, radiation dose, pitch, slice thickness and airway orientation with respect to the scanner influence spatial resolution. Measurements are further affected after acquisition by the reconstruction algorithm used, window settings, segmentation algorithm and the method of dimension analysis. Small airways (diameter $<2 \mathrm{~mm}$ ), and particularly the terminal bronchioles (diameters $<0.2 \mathrm{~mm}$ ), may be involved in obstructive airways diseases [12-14]. Although some groups have reported measuring airways down to $0.5 \mathrm{~mm}$ in diameter $[15,16]$, accurate and reproducible measurements have only been demonstrated consistently in airways down to $2 \mathrm{~mm}$ in diameter [9, 17-20]. Despite this limitation, NAKANO et al. [21] showed that the wall area in intermediate-sized airways measured with HRCT in COPD patients correlates with the wall area in small airways measured histologically. These findings suggest that changes in the intermediate-sized airways, which can be reliably measured, reflect pathology in the smaller airways. Novel software solutions allow for "sub-voxel" measurements from MDCT-derived data [22, 23] but cannot improve image resolution beyond the smallest voxel size.

\section{Window width and level}

CT images reflect the density of lung tissue in Hounsfield units $(\mathrm{HU})$ and are displayed in 16-bit grayscale. The density of human tissue lies between -1000 HU representing air (black) and +1000 HU representing cortical bone (white; fig. 1). The window width is the range spanned by the grayscale and the window level is the Hounsfield unit at the centre of this range. Altering the width and level on a CT display appears to "increase" or "decrease" airway size and can affect measurements. Several groups have investigated optimal window settings for quantitative airway measurements [19, 24-27]. While most studies in phantoms have shown that window level and not width determines the accuracy with which the airway lumen [19, 27] and wall area [25] are measured, BANKIER et al. [24], using inflation-fixed cadaveric lungs, found window width rather than level affected this accuracy. This suggests that phantom studies may not reflect in vivo conditions in which partial volume averaging plays an important role.

\section{Measuring airway dimensions from CT data}

Beyond the technical issues related to acquisition of CT data, other factors affect airway measurement accuracy. These include the method used to quantify the airway dimension from the image and the relationship between the measured 


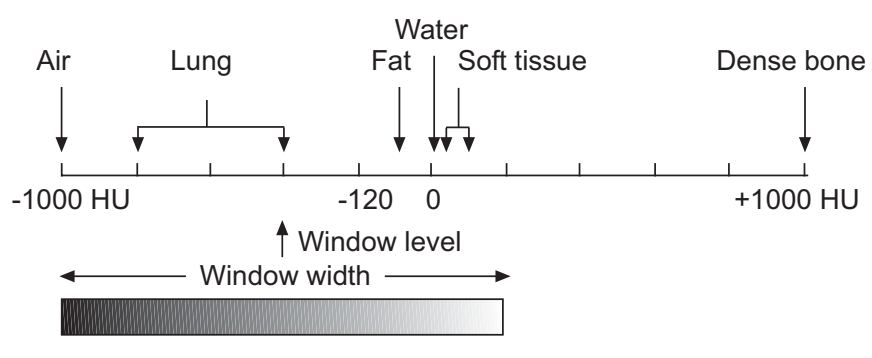

FIGURE 1. The relationship between window width, level and the typical density ranges of human tissues as imaged using a modern computed tomography scanner.

airway and the axis of the CT scanner: a relationship central to the notion of partial volume averaging. This is best defined as the inaccurate representation of airway wall thickness and lumen area resulting from more than one tissue type (airway wall, mucosal secretions and air, etc.) being incorporated into a single voxel (see below).

Initial attempts to measure lumen and wall area used manual tracing of the inner and outer wall perimeter from enlarged CT images [19]. Aside from its subjective and labour-intensive nature, manual tracing has been shown to systematically overestimate airway wall thickness and underestimate the airway lumen [18]. Subsequently, automated and semi-automated computer-based algorithms have been developed. The principles underlying these techniques include: threshold-based methods [18, 28, 29]; the full-width at half maximum (FWHM) distance [20, 30]; score-guided erosion [18]; "Laplacian of Gaussian" filters to detect airway edges [9,31]; and algorithms based on idealised airway models [32, 33]. These computer algorithms are based on tissue density measures rather than on arbitrary grayscale measures and have been discussed elsewhere in detail $[17,34,35]$. The FWHM method has been widely used to investigate airway changes in obstructive lung diseases $[20,21,36]$. However, this method systematically overestimates wall area and underestimates lumen area [21, 32]. Advanced software algorithms, taking into account the point spread function of the scanner and airway centre lines, and using applications such as optimal surface feature finding and graph search functions are now being developed and significantly improve the robustness of lumen area measurements [23].

The angle of orientation at which an airway is imaged relative to the scan plane can also affect dimension measurement. Any deviation from an orthogonal plane (i.e., when the airway is perpendicular to the CT gantry) increases partial volume averaging. Partial volume averaging arises when a single voxel contains more than one tissue type, for example, a voxel near an airway wall which may contain both air and wall. In such a case, the voxel's grayscale value reflects the mean weighted average of the different tissue densities [37]. Partial volume averaging leads to overestimation of airway wall thickness and underestimation of airway lumen. It occurs in all airways, increases with scanning angle and is most pronounced in airways imaged parallel to the scanner, since in this plane more voxels contain a mixture of airway lumen and wall. It is linearly related to airway size [8]: while a greater absolute error is seen in larger airways a greater relative error occurs in small airways which have a higher wall-to-lumen ratio.

Slice thickness is another important determinant of partial volume averaging. As CT slices are two-dimensional images representing three-dimensional data it follows that, when airways are imaged at angles other than $90^{\circ}$, more wall area, derived from the $z$ axis, is incorporated into the $x$ and $y$ dimensions leading to an apparent increase in wall thickness and decrease in lumen area (fig. 2) [24]. Thinner slices and more orthogonal airway scanning therefore lessen the degree of partial voluming averaging $[9,27]$.

To limit the affects of partial volume averaging on measurement accuracy, many studies have only measured airways that lie orthogonal to the scanner plane $[19,26,28-30,38]$. However, this excludes a majority of airways. A superior approach is to either correct the angle mathematically [18, 39] or reconstruct the data in such a way as to "re-orient" the slice to be perpendicular to the airway. "Skeletonisation" facilitates such reconstruction by segmenting the bronchial tree and connecting the resulting contiguous airway centrelines to form a branching tree-like structure (fig. 3) [22, 31, 36, 40]. Creation of an airway skeleton facilitates orderly exploration of the tracheobronchial tree and serves as a viewpoint trajectory for navigation during bronchoscopy [22]. Although skeletonisation limits the effect of partial volume averaging, it does not eliminate it altogether, especially in CT scanners where the voxels are not isotropic (i.e. of equal size in the $x, y$ and $z$ axes). Other limitations include "leakage" of the airway into extraluminal regions, inadvertent addition or omission of tree branches and imaging artefacts.

\section{Three-dimensional airway visualisation and virtual bronchoscopy}

There are two main ways of visualising airways in three dimensions by reconstruction from volumetric datasets. External rendering, analogous to CT bronchography, depicts the external surface of the bronchial tree, whereas internal
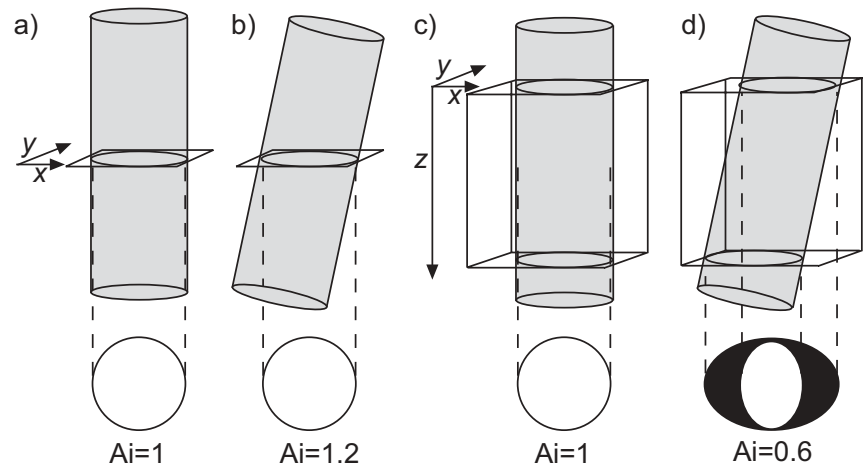

FIGURE 2. a) Theoretical cross-sectional area (Ai) resulting from transection of a cylinder at right angles. b) Ai increases if the cylinder is cut obliquely, a result of increased long-axis diameter. c) During computed tomography imaging, partial volume averaging results as slice thickness (the $z$ axis) is incorporated. d) Unless the airway is oriented perpendicular to the $z$ axis of the scanner, an apparent increased wall thickness and reduced relative Ai results as data from the voxel $z$ axis is averaged to produce the final two-dimensional image. Reproduced and modified from [8] with permission from the publisher. 
a)

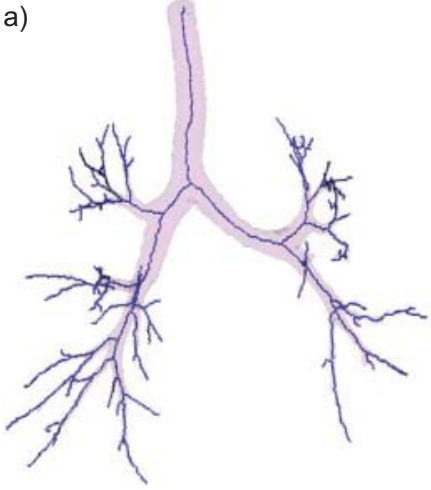

b)

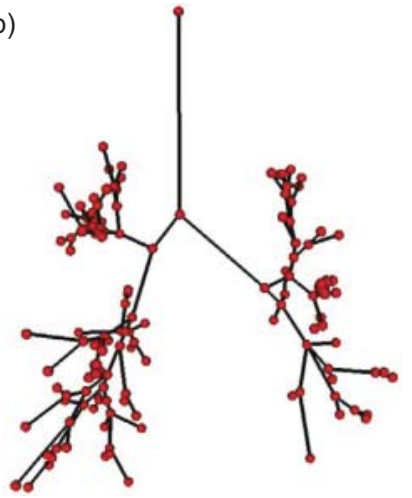

FIGURE 3. a) Skeletonisation of a human airway tree showing the centrelines. b) The corresponding formal tree structure, in which a path between two branch points is replaced by a single edge. Reproduced from [22] with permission of the publisher.

rendering, better known as virtual bronchoscopy, depicts the internal surface of the bronchial tree, allowing navigation of the tree "endoscopically". Both provide clinically useful images and allow qualitative assessment of airway shape and, in the case of external rendering, relationship to adjacent structures [41]. External rendering allows assessment of stenosis length to guide pre-operative stent selection [42, 43].

Virtual bronchoscopy performs well in comparison with fibreoptic bronchoscopy in detecting airway stenoses in adults $[44,45]$ and children [46] but may overestimate the number of stenoses, particularly in segmental airways [44]. It is a useful tool for noninvasively grading stenotic lesions [43, 44, 47, 48], and has several advantages over conventional bronchoscopy, allowing a view of the airway from below to "look up" at the vocal cords and provides views of airways beyond tight obstructing lesions in regions which bronchoscopes cannot access. Dynamic airway narrowing, as seen with bronchomalacia, may be missed on single-phase internal rendered scanning $[46,49]$ and is more reliably detected by comparing paired inspiratory and expiratory scans or using cine-CT during dynamic forced expiratory manoeuvres [50-53].

In summary, the following can be concluded regarding CTbased measurement techniques: 1) automated systems reduce subjectivity and improve the speed, reproducibility and accuracy over manual tracing; 2) measurements in airways as small as $2 \mathrm{~mm}$ are highly accurate and precise, and measurements in airways smaller than this are improving with modern scanners and software algorithms; 3) partial volume averaging effects can be reduced (but not eliminated) by using thinner slices, imaging airways orthogonal to the CT scanner axis or using isotropic voxel scanners, and; 4) skeletonisation algorithms facilitate the reconstruction of CT data orthogonal to the airway axis reducing the effects of partial volume averaging and aiding navigation through the tracheobronchial tree.

\section{VIDEOBRONCHOSCOPY}

With interventional bronchoscopies becoming increasingly common and an expanding array of indications and procedures now available, there is a growing need for bronchoscopy to provide more quantitative information in real time. Since the first reports of tracheobronchial stenting by DUMON [54] and others $[55,56]$ in the 1980s, bronchoscopists have strived to overcome complications such as stent migration or erosion into adjacent structures resulting from suboptimal stent size [57, 58]. Acquiring quantitative airway dimensions during a procedure appeals to bronchoscopists, who must assess airway calibre, monitor disease progression, determine treatment and its effectiveness with time, and choose the appropriate size of endobronchial stents or valves. In the setting of airway obstruction, bronchoscopy is aided by pre-procedure virtual images from MDCT, especially if the stenosis is too narrow for the bronchoscope to traverse. However, it is only during bronchoscopy that blood clot, secretions and necrotic tissue can be distinguished and tumour vascularity assessed. Also, if dilatation or endoscopic tumour resection have been attempted, local anatomy may be altered. Thus, it remains important for the bronchoscopist to be able to confirm the airway dimension immediately prior to stent deployment. However, several factors limit the ease with which bronchoscope-based measurements are made. These are discussed below.

\section{Technical aspects of quantitative measurement from bronchoscopy}

Distortion from the "wide-angle" or "fisheye" lens at the tip of the bronchoscope limits measurement accuracy from the field of view (FOV; fig. 4). The lens increases the FOV produced by the small camera but the trade-off is "distance" and "radial" image distortion.

\section{Distance distortion}

This occurs as the distance between an object and the lens is increased. Optimal lens-to-object distance varies according to the optics of each bronchoscope. DoOLIN and STRANDE [61] showed that, when using a paediatric 4-mm rigid bronchoscope, a distance of $15 \mathrm{~mm}$ provided the least variation of cross-sectional area from the actual size. Subsequent studies using adult flexible bronchoscopes have tended to be performed at this distance $[59,62]$. A range of $10-40 \mathrm{~mm}$ has been shown to be most accurate with a flexible paediatric bronchoscope [63] and 5-20 mm with an adult bronchoscope [64]. At distances $>20 \mathrm{~mm}$, an object's size relative to the FOV
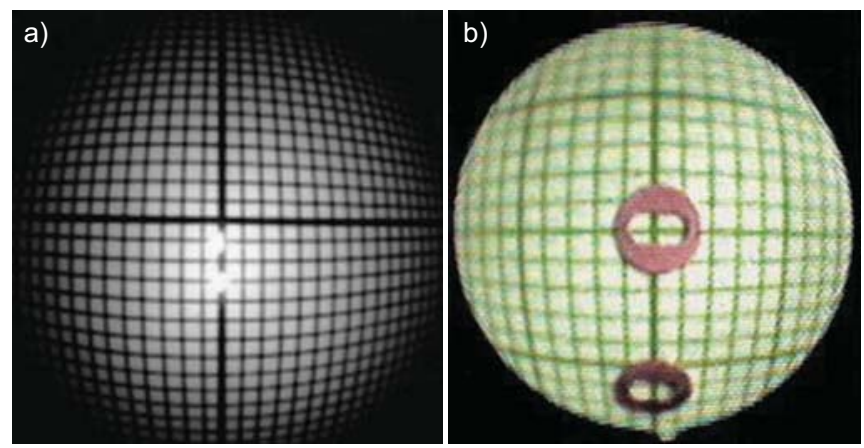

FIGURE 4. a) Distorted image of millimetre-grid paper captured using a video bronchoscope (BF 1 T 200; Olympus, Tokyo, Japan). b) Circular object in the centre of a bronchoscope's field of view and its apparent size reduction and ellipsoid distortion when viewed at the image periphery. Reproduced from [59] and [60] with permission from the publisher. 
begins to plateau and there is a reduced ability to detect the edge of an object, thus limiting ease of quantification [64]. Knowing the distance from the bronchoscope tip to an object is also required to convert pixel area to absolute area, unless a calibration target is placed in the FOV.

\section{Radial distortion}

Radial distortion [64], also known as barrel [65] or spatial distortion [59], refers to the nonlinear, morphological changes in image size and shape which occur as an object moves from the centre to the periphery of the FOV (fig. 4b). As the object approaches the periphery, its size diminishes, circular shapes appear more ellipsoid and straight lines appear curved. Within the central $40 \%$ of the bronchoscopic FOV radius, the mean decrease in image size is only $12 \%$, hence distortion within this area may not require correction [64].

\section{Overcoming distortion effects}

To quantify images in the field of view both types of distortion must be addressed. Distance distortion can be avoided by keeping the tip of the bronchoscope within an appropriate working distance from the object (see above). Radial distortion can be corrected by numerically processing the image using either an analytic approach [60-62, 64] or by using coordinate transformation $[59,66]$, in which a distortion correction matrix is generated by comparing multiple points on a distorted image with the same points on an undistorted image.

It is technically easier to overcome the effects of distortion using ex vivo or bench-top techniques than during in vivo human bronchoscopy. In explanted pig airways, MitCHELL and SPARROW [67] quantified lumen diameter and area using digital still images of a stained mucosa-lumen interface obtained through a rigid bronchoscope. Radial distortion was avoided by using a nondistorting lens and calibration was achieved by passing a calibration target into the FOV.

In a study using a 5-mm rigid bronchoscope, morphometric image correction was examined [61]. Mathematical image analysis was used to correct radial lens distortion of images of grid paper. Significantly reduced errors of circle area measurement from 17.6 to $4.3 \%(\mathrm{p}<0.003)$ were reported [61]. This analysis technique was then used to measure the crosssectional area of large airways dissected from two pigs [62] The measured area and short-axis diameter correlated well with digitised photos in airways of cross-sectional area $<80 \mathrm{~mm}^{2}$ and approximately $<10 \mathrm{~mm}$ in diameter $(\mathrm{r}=0.94)$ but not in larger airways $(r=0.58)$. This reflects the increased distortion at the periphery of the FOV. Other limitations of this method include: the inability to display measurements in real time; the need to measure the distance between the tip of the bronchoscope and object for calibration, a difficult task in vivo; the requirement for a different correction algorithm for each bronchoscope; and measurement correction from only a single image at a time.

MCFAWN et al. [60], observing that lens distortion is regular and uniform, developed an algorithm using polynomials to correct the position of each individual pixel in the FOV. This method is not affected by object size or shape and lens correction is independent of the distance between the bronchoscope and the object. Third order polynomial functions produced the greatest accuracy of area measurements, reducing the area measurement error of a peripherally located square on grid paper from $42 \pm 4.2 \%$ to $2.9 \pm 0.97 \%$ after correction. The lack of clinical uptake for this otherwise simple and accurate method could be explained by the requirement for a calibration marker in the FOV as well as the lens-specific and image-by-image nature of measurement correction failing to provide real-time measurements.

\section{Measuring distance between the bronchoscope and region of interest}

Measuring the distance between the tip of the bronchoscope and any given site in vivo is usually performed by advancing the tip of the bronchoscope to the site of interest then withdrawing the scope a specified distance from the patient's teeth or lips. This is prone to inaccuracy given the constantly changing spatial relationship between the bronchoscope tip and region of interest due to respiration and cardiac motion and the often inadequate measure of bronchoscopic translation. Techniques have been developed to enable better measurement of this distance.

DORFFEL et al. [59] projected a circular beam of light onto the endobronchial surface using a low intensity Nd:YAG (neodymium-doped yttrium aluminium garnet) laser reflected off an angled mirror $15 \mathrm{~mm}$ from the bronchoscope tip (fig. 5). Radial distortion was corrected via custom-made image processing using coordinate transformation. Measured areas of polystyrene tubes correlated closely with the known diameters with an intraclass correlation coefficient (ICC) 0.97 $(p<0.01)$. Validation in three explanted pig tracheas also showed good area correlation with digitised photomicrographs at the same sites (ICC 0.87, p<0.01). The technique appeared useful during in vivo measurements in four patients with tracheal stenosis when compared with helical CT. The stenotic area was overestimated by $15 \mathrm{~mm}^{2}(14 \%)$ in one patient and underestimated in another by $10 \mathrm{~mm}^{2}(34 \%)$, although the small sample size limited statistical analysis.

If a calibration marker is placed at a site of interest, it is not necessary to know the precise distance from the bronchoscope to that site [60]. CZAJA et al. [68] used a special measuring device with a bendable arm, which they passed through a bronchoscope adjacent to a lumen of interest (fig. 6). A line perpendicular to the bronchial wall was manually drawn on the bronchoscopic image

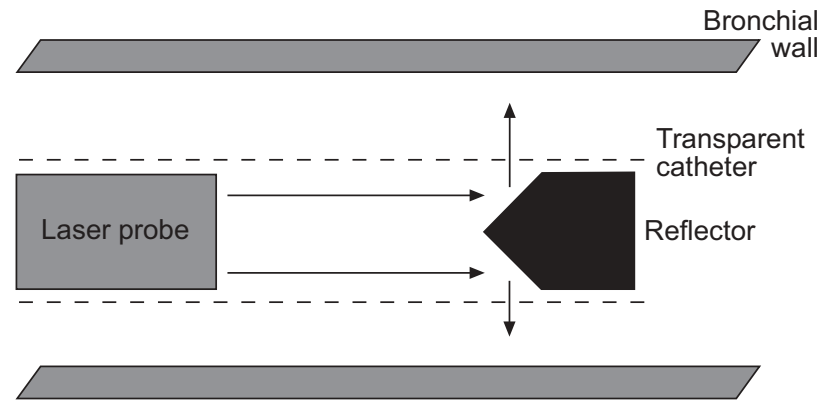

FIGURE 5. Low intensity Nd:YAG (neodymium-doped yttrium aluminium garnet) laser reflecting a rim of light (arrows) at a known distance from the bronchoscope on the airway wall. Reproduced from [59] with permission from the publisher. 
and a computer program (details not specified) determined diameter, perimeter and cross-sectional area. Diameters at airway orifices were compared with CT measurements in airways ranging $2-23 \mathrm{~mm}$ diameter in 40 patients. Bland-Altman comparisons showed close agreement (mean diameter difference across all airways measured $0.071 \mathrm{~mm}$, limits of agreement $-1.071-0.929 \mathrm{~mm}$ ). Unfortunately, this study did not correct for lung volume: CT image acquisition was near total lung capacity, but during bronchoscopy dimensions are measured closer to functional residual capacity for which smaller airway calibre would be expected. Furthermore, the investigators did not conduct repeatability measurements, which is of concern given the subjective nature of lumen identification. Again, the calculations were conducted "off line" rather than in real time.

\section{Colour-adjusted calibration techniques}

A recent quantitative videobronchoscopy approach [69] combines lens magnification correction [63] with the "colour histogram mode technique" (CHMT) using the National
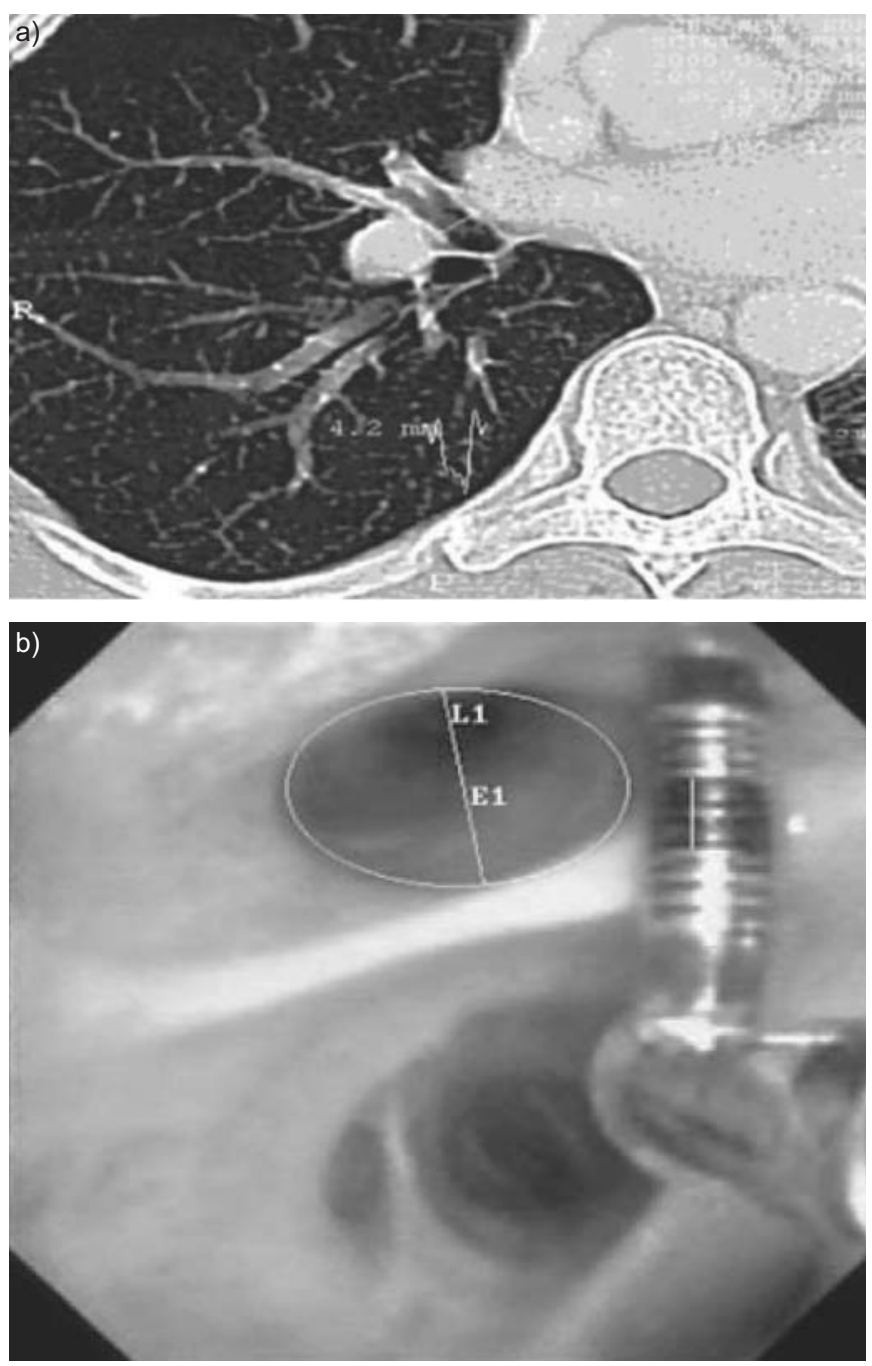

FIGURE 6. Measurement of the right middle lobe bronchus. a) Computed tomography and b) quantitative videobronchoscopy with measuring device in the lumen. Reproduced from [68] with permission from the publisher.
Institute of Health (Bethesda, MD, USA) software "ImageJ" [70]. CHMT offers the potential for in vivo, "near" real-time airway calibre measurements. This technique was used to assess bronchomalacia longitudinally in children demonstrating similar rates of change of cross-sectional area in normal and abnormal sites over a 1-yr period [71]. Briefly, CHMT displays colours from a bronchoscopic image in histogram format and a user adjusts the display via the ImageJ colour balance function (fig. 7). Brightness is adjusted such that structures darker than the brightness mode point are shaded black (e.g. the airway lumen), while regions of the airway proximal to the mode point appear lighter and are shaded yellow, red and white depending on how much light is reflected from the tissue. The airway lumen can then be automatically traced from this image and superimposed on the original bronchoscopic view. Using lens magnification correction [63], lumen dimensions can be calculated. Validation studies in children showed strong intra- and inter-observer repeatability when each image was analysed with CHMT but did not include repeat acquisition of images at the same site or by different examiners. Limitations also include the need to measure the distance between the site of interest and the bronchoscope and the unknown affect on tissue reflectance of various physiological conditions, such as oxygen desaturation and anaemia. Human CT correlation has not been performed.

There are several reasons why, despite these techniques, quantitative videobronchoscopy has not yet entered routine clinical practice. First, no current method provides measurements at the time of viewing. Even those techniques offering "near" real-time analysis [68, 69] require image processing, limiting the ability to provide the proceduralist with timely information. This has obvious implications for procedures such as stenting, where dimensions are needed immediately. Secondly, the distortion inherent in the bronchoscope lens optics limits the ease with which measurements can be made. Correcting this distortion can be complex, lens specific and time consuming. Thirdly, the need for calibration targets placed in the field of view further complicates procedures.

Newer techniques may overcome some of these limitations. Linking the aforementioned technologies, bronchoscopic images can now be merged with MDCT datasets [72, 73]. In this process, the two-dimensional videobronchoscopic image is first converted to a three-dimensional airway tree using "shape

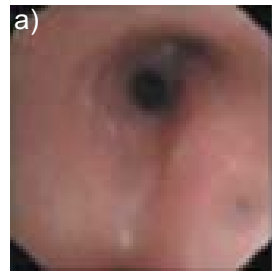

Original image

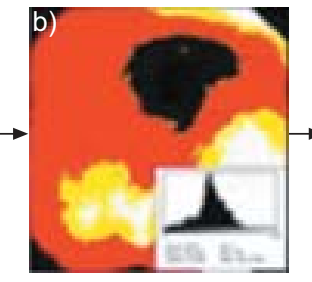

Colour adjusted and histogram

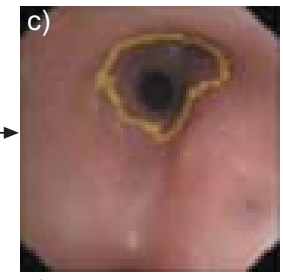

Original and outline that is measured
FIGURE 7. Transitions in the image created by the process of dividing the colour scales of the image around the mode point of the histogram, as shown in the insert. The final (right) image shows the area to be measured superimposed on the actual image. The image shown is the right main stem bronchus. Reproduced from [69] with permission of the publisher. 
from shading algorithms" while bronchoscopic lens distortion is corrected using a least squares approach [66]. These data are then merged with the virtual bronchoscopy images derived from MDCT. The resulting comprehensive synergistic datasets produce a virtual bronchoscopic image in true colour from which airway dimensions can be accurately measured and subtle mucosal colour changes assessed.

\section{EMERGING TECHNOLOGIES}

Some newer technologies have the capacity to provide quantitative assessment of airway dimensions in vivo. Appreciating the distinctions between current and future developments is increasingly difficult as they cross clinical, research and engineering disciplines (table 3).

\section{Confocal fluorescence endomicroscopy}

Confocal microscopy is a light microscopy technique with enhanced optical sectioning beyond that of conventional microscopy, which is achieved through the use of a scanned point beam and a pinhole aperture to eliminate out-of-focus light [74]. Confocal fluorescence endomicroscopy takes conventional confocal fluorescence microscopy to the next level by combining it with flexible fibreoptic technology in one of two ways [75]. In fibreoptic confocal fluorescence endomicroscopy (FCFM), the microscope objective of a confocal unit is replaced with a fibreoptic bundle miniprobe that is passed through the working channel of a bronchoscope. The use of a bundle facilitates miniaturisation of the probe at the expense of image pixelation, since the number of fibres in a bundle is limited. A current FCFM model [76] detects light emitted by endogenous fluorophores (molecules present in tissue which, when excited by light at a particular wavelength, emit light at a longer wavelength) in the superficial layers of the subepithelial bronchial wall. Exogenous fluorophores, such as fluoroscein, could potentially be inhaled or administered intravenously to further improve resolution or detect dysplastic tissue. Realtime scanning of the bronchial surface produces a highresolution "optical biopsy" to a depth of $50 \mu \mathrm{m}$. An alternative approach is to use a single-mode optical fibre (rather than a bundle), which serves as an emitter and confocal receiver of light, while the microscope objective is positioned at the distal tip of the bronchoscope. This design improves spatial resolution but limits the degree of miniaturisation.

THIBERVILLE et al. [76] outlined early findings of FCFM in healthy controls and patients with pre-malignant and benign airway pathology. They found five distinct and reproducible microscopic patterns of basement membrane architecture which were distorted in diseased states. This technology has potential applications in the study of basement membrane changes in obstructive lung diseases, which would allow not only quantitative assessment of membrane thickness, but also detection of qualitative changes that might prove characteristic of pathological or even clinical disease phenotypes. Furthermore, the Confocal Miniprobe ${ }^{\mathrm{TM}}$ (Mauna Kea Technologies, Paris, France) can image terminal airways and even alveoli, so-called Alveoscopy $^{\mathrm{TM}}$, potentially opening the way for in vivo quantitative assessment. Despite the potential, an important limitation of confocal endomicrosopy is the tiny field of view (up to $600 \mu \mathrm{m}$ ). Although image reconstruction mosaicing techniques may, in part, ameliorate this by co-registering images as the probe moves [77] to create a larger overall picture, the question arises as to how many sites would need to be imaged to provide a representative picture. Similarly, the depth of tissue penetration is small compared with other endoscopic techniques, meaning only superficial structures in the wall can be visualised. Finally, most probes are "forward looking" and, thus, not ideal for imaging airway walls.

\section{Optical coherence tomography}

Optical coherence tomography (OCT) is a light microscopy technique with enhanced "optical sectioning" (effective resolution in depth) beyond that of conventional microscopy [78]. It employs broadband, near-infrared light with limited (temporal) coherence, and uses optical interferometry to measure delays (and distance) between a reference light beam and incident light reflected from tissue structures. In its endoscopic realisation, a light beam from a single-mode optical fibre is directed onto a tissue. Beam scanning may be achieved in various ways, but a common means is through a rotating probe $[79,80]$. OCT produces images of high resolution (approaching that of histology) with tissue penetration to 1-3 $\mathrm{mm}$ and can, therefore, perform an "optical biopsy" of subsurface architecture.

OCT is well established in the field of ophthalmology (retinal imaging) [81, 82] and is emerging as a diagnostic tool in cardiology and gastroenterology [79, 83-87]. Its current use in the respiratory system is limited: research to date has focused on detection of dysplastic and neoplastic epithelial changes. Airway validation studies using explanted animal and human airways have been performed and demonstrate near-histological

TABLE 3 Comparison of available and emerging quantitative airway imaging modalities

\begin{tabular}{lccccc} 
Technique & Availability & Radiation & Dynamic views & Resolution & Current use \\
\hline Bronchoscopy & Wide & No & Yes & + & Clinical \\
MDCT & Wide & Yes & In cine mode & ++ Clinical/research \\
EBUS & Increasing & No & Clinical & ++ & Research \\
MRI & Limited & No & Possible & + & Research \\
OCT & Very limited & No & Yes & +++ & Research \\
FCFM & Very limited & No & Yes & ++++ & r+ \\
\hline
\end{tabular}

MDCT: multidetector computed tomography; EBUS: endobronchial ultrasound; MRI: magnetic resonance imaging; OCT: optical coherence tomography; FCFM fibreoptic confocal fluorescence endomicroscopy. 
resolution [88-90]. A recent study by CoxsON et al. [91] used OCT to measure airway lumen and wall area in 44 current and former smokers. The measurements correlated strongly with those of HRCT but were lower by $31 \%$ and $66 \%$ for lumen area and wall area, respectively, reflecting the unmatched lung volumes in this study, i.e. total lung capacity during the CT acquisition and conscious sedation for the OCT acquisition. The high-resolution small airway images produced in the study suggest an important future role in studies assessing small airway remodelling (fig. 8).

For adequate subsurface tissue imaging, the OCT probe must, in general, be positioned in close proximity to the airway wall, often in contact. This approach tends to limit the field of view to a small fraction of the overall airway circumference. A modification by YUN et al. [87], termed optical frequencydomain imaging, enabled the probe to be positioned up to $7.3 \mathrm{~mm}$ from the tissue wall. By combining high-speed probe rotation with axial translation, this technique is capable of high-speed acquisition of volumetric data of subsurface morphology and has been mainly directed towards cardiovascular imaging. While promising as a tool for quantifying airway dimensions, a balloon sheath is presently required to stabilise the device within the lumen, potentially distorting airway anatomy and obstructing breathing.

Another adaptation, referred to as anatomical OCT (aOCT), extends the axial scanning distance of conventional subsurface imaging OCT and concentrates on characterising internal anatomy, i.e. lumen internal shape and size. To do this, the range of axial scanning distance is increased up to $36 \mathrm{~mm}$ without the requirement of a balloon sheath, allowing hollow organs with diameters up to $72 \mathrm{~mm}$ to be imaged [92, 93]. Transverse resolution is reduced, as is the signal from subsurface structures, but the entire airway circumference can be imaged in cross section. Our group have validated aOCT in the upper airway using simultaneous CT scanning [94] and used aOCT for the assessment of airway size and shape in obstructive sleep apnoea $[95,96]$. The aOCT probe can be passed into the lower airway through the working channel of a conventional bronchoscope to a region of interest. Upon
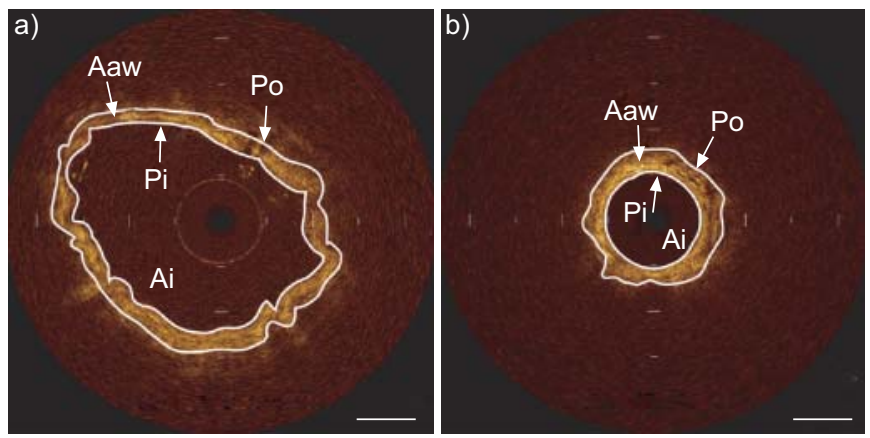

FIGURE 8. Optical coherence tomography images of a third- (a), and fifthgeneration airway (b). The internal perimeter (Pi) and outer perimeter (Po) of the airway wall were manually traced using ImageJ software (National Institute of Health, Bethesda, MD, USA), and the lumen area (Ai) and wall area (Aaw) were calculated using these boundaries. Scale bars $=2 \mathrm{~mm}$. Reproduced from [91] with permission from the publisher. rotation of the probe, a two-dimensional cross section of the airway is built up in much the same way as a radar image. With mechanical retraction of the probe, a three-dimensional volume dataset can also be constructed [97]. aOCT could prove to be a powerful tool for the interventional bronchoscopist during placement of endobronchial stents using realtime measured stenotic dimensions to guide stent selection. Several limitations of other imaging modalities are addressed by aOCT. For example, as the probe follows the course of the airways, the measured cross section at any point is likely to be orthogonal to the airway wall, thereby limiting partial volume averaging. Another advantage of aOCT is the ability to image in real time, providing dynamic as well as structural detail. Finally, there is no lens distortion and no calibration marker is needed.

\section{Magnetic resonance airway imaging}

Until recently, magnetic resonance imaging (MRI) has been largely in the domain of neurology and musculoskeletal medicine. MRI images of airways and lung parenchyma have generally been of poor quality, owing to the limited MRI signal from lung tissue caused by its heterogeneity and the lack of protons (low water content) in the air spaces [98]. Respiratory motion artefact also reduces image quality as acquisition time exceeds a comfortable breath hold, necessitating respiratorygating techniques. Despite these limitations, MRI has appeal, in particular the ability to provide functional information in the absence of radiation.

Hyperpolarised gases, a new class of MRI "contrast agent", overcome the limitations of imaging lung tissue [99]. These are inert gases, such as ${ }^{3} \mathrm{He}$ or ${ }^{129} \mathrm{Xe}$, whose nuclei have been hyperpolarised by optical excitation using polarised lasers. In this state of increased energy, the gas has an enhanced MRI signal relative to its unpolarised state. Following inhalation, ${ }^{3} \mathrm{He}$ emits sufficient signal within the airspaces to be detected by MRI, producing images at high spatial resolution [98]. Scanning commences just prior to gas inhalation and continues through the breath. This avoids the obscured images which occur as gas enters the distal airspaces [100]. Unfortunately, the constantly changing lung volume makes direct comparison with other imaging modalities difficult.

Using dynamic hyperpolarised ${ }^{3} \mathrm{He}$, LEwIS et al. [101] quantified airway diameters of bronchial tree generations $0-5$. They compared diameter measurements at each airway generation with the theoretical diameter based on the Weibel lung model. In airways ranging $3-18 \mathrm{~mm}$ diameter, they found no significant difference between the MRI measurements and the predicted airway dimensions. This validation falls short of the rigorous methods described in the previous MDCT/HRCT and videobronchoscopy studies [8, 9, 18, 20, 21, 39], but demonstrates the potential for MRI to measure the central airways.

To improve the applications of MRI imaging, slice thickness will need to be reduced $(13 \mathrm{~mm}$ in the study by Lewis et al. [101]), the effect on measurement accuracy of scanning at various angles will need to be assessed, and the thresholdbased measurement algorithm will require further validation. An ongoing problem remains access to MRI and hyperpolarised gases. These gases are expensive, only manufactured 
by several specialised units and must be transported and used within hours of production.

\section{Endobronchial ultrasound}

Transthoracic ultrasound does not adequately visualise the intrathoracic airways. Only extrathoracic airway structures, such as the subglottic trachea, can be measured [102]. Endoscopic ultrasound, originally developed for gastrointestinal assessment in the 1980s, has been adapted to the bronchoscope and today, linear array endobronchial ultrasound (EBUS), is widely used for lung cancer diagnosis and staging.

The $20 \mathrm{MHz}$ EBUS radial probe, as distinct from linear array EBUS, is a thin ultrasound transducer that can be passed through a bronchoscope biopsy channel into an airway of interest. Rotation of the transducer generates a cross-sectional acoustic image of the airway. As acoustic attenuation precludes effective ultrasound wave penetration through air, a saline-filled sheath is inflated around the transducer to provide sonic coupling with the airway wall. Under these circumstances, endoscopic ultrasound penetrates the airway wall up to a depth of $2 \mathrm{~cm}$, making it suitable for morphological assessment of both lumen diameter, area and wall thickness [103].

SHAW et al. [104] imaged the posterobasal segment of the right lower lobe in 12 healthy volunteers, measuring the ratio of wall thickness to diameter as well as the wall area percentage, and found a close correlation with measurements derived using HRCT. However, lung volume was not standardised in the study, possibly explaining why the average internal diameter measured in sedated patients by EBUS was less (albeit not significantly) than that measured with HRCT scanning at total lung capacity $(4.9 \pm 0.9 \mathrm{~mm}$ versus $5.2 \pm 1.0 \mathrm{~mm})$. The authors also assessed the effect of the saline-filled sheath on diameter and wall thickness in explanted sheep airways with and without the sheath inflated. Close concordance was demonstrated in this ex vivo study, although the influence of inflating a sheath in vivo on airway size, shape and mucosal folds has not been assessed.

\section{FUTURE APPLICATIONS OF QUANTITATIVE AIRWAY IMAGING \\ Cystic fibrosis and bronchiectasis}

Cystic fibrosis lung disease is characterised by airway dilatation and wall thickening, mucosal plugs and gas trapping. Several scoring systems based on HRCT findings have been developed to assess disease severity. While these scores correlate with lung function $[105,106]$ they can detect disease progression while lung function is stable [107] or even normal [108]. Regular scans may be performed to monitor disease progression and determine the timing of intervention, although this increases the lifetime risk of radiation-induced cancers by up to $13 \%[109,110]$. Low-dose protocols and reducing the number of slices per scan limit exposure but lessen test sensitivity [111-113]. Endoscopic imaging coupled with OCT, confocal microscopy or EBUS offers the opportunity to detect changes of airway wall thickening and remodelling in cystic fibrosis, possibly with greater sensitivity than MDCT. It is possible that, instead of regular CT scans, regular bronchoscopies could be performed to monitor disease progression using the emerging techniques. This approach, in place of, or complementary to, CT scoring, could enhance early disease detection while limiting radiation exposure, although the risks of repeated bronchoscopy versus CT warrant consideration.

\section{Asthma}

MDCT analysis of asthmatic airways provides a continuing source linking structure to function [34]. Measurements of wall area, lumen area and airway calibre will continue to be used in research studies to define pathology and elucidate mechanisms of excessive airway narrowing while newer applications of MDCT, including the linkage of parenchymal regions of low attenuation to airway changes, will expand the research applications of this modality [114-116]. Endoscopic imaging techniques, in particular confocal microscopy and OCT, offer the potential to complement MDCT by providing higher resolution detail of airway wall structure in lieu of histology. For example, heterogeneity of airway narrowing, a feature demonstrated by MDCT following exposure to vasoconstricting agents, could be assessed during bronchoscopy by applying a localised agent and simultaneously measuring real-time airway calibre and wall thickness. This could be repeated at several locations and on separate occasions.

\section{COPD}

COPD is characterised by airflow obstruction that is not fully reversible. It is associated with CT evidence of pathological changes in the central and small airways, as well as areas of low attenuation in the lung parenchyma [117-119]. Recent attention has focused on phenotyping COPD into distinct subgroups based on MDCT features [120]. These include "emphysema predominant", "airway wall remodelling (thickening) predominant" and a subgroup containing features of both. For a given phenotype, patients may exhibit different clinical characteristics and may respond to different treatment strategies [20, 91, 119, 121]. The emerging endoscopic techniques could extend this phenotypic differentiation by further identifying and characterising the nature and distribution of wall thickness and lumen area changes with the ultimate goal of accurately evaluating disease progression and individualising treatments to target these phenotypes. As with asthma, the relationship between parenchymal areas of low attenuation assessed by MDCT, with airway pathology measured by conventional methods (e.g. spirometry, lung volumes, bronchial challenge and exercise tests) appears important [20, 122,123 ] and the advent of three-dimensional MDCT datasets with semi-automatic analysis algorithms for volumetric analysis will allow further characterisation of regional hyperinflation and expiratory airflow obstruction [124].

\section{CONCLUSIONS AND FUTURE DIRECTIONS}

There is an expanding array of tools with which to measure lower airway dimensions, with benefits for pulmonologists, interventional bronchoscopists, researchers and, ultimately, patients. Rather than competing, in many senses they add complementary information about a complex structure, with no single tool suitable for all settings. For a given need, the airway size, required accuracy, local availability and clinical setting interact to determine the most suitable tool. The bronchoscopist, more interested in larger airways, may be willing to forego sub-millimetre accuracy in preference for real-time imaging and ease of repeat measurements directly 
following a procedure. Conversely, researchers wishing to evaluate airway dimensions with a higher degree of accuracy to gain insights into remodelling and inflammation will stand to benefit most from the newer MDCT reconstruction and measurement algorithms including the use of fused MDCT/ videobronchoscopy datasets as they are further developed and refined. Emerging technologies, such as endoscopic OCT and confocal endomicroscopy, provide new avenues for airway imaging, acquiring dynamic as well as structural data at unprecedented resolution.

\section{SUPPORT STATEMENT}

Supported by National Health and Medical Research Council of Australia (NHMRC) Research Project Grant No. 513854 and NHMRC Postgraduate Research Scholarship No. 463926 (J.P. Williamson), and NHMRC Senior Research Fellowship No. 513704 (P.R. Eastwood).

\section{STATEMENT OF INTEREST}

None declared.

\section{ACKNOWLEDGEMENTS}

The authors wish to thank N. Hicks and P. Muir of the Dept of Radiology, Sir Charles Gairdner Hospital, Perth, Australia for their technical review of the manuscript.

\section{REFERENCES}

1 Hurter T, Hanrath P. Endobronchial sonography: feasibility and preliminary results. Thorax 1992; 47: 565-567.

2 Weibel E, Gomez D. Architecture of the human lung. Use of quantitative methods establishes fundamental relations between size and number of lung structures. Science 1962; 137: 577-585.

3 Boyden EA. A synthesis of the prevailing patterns of the bronchopulmonary segments in the light of their variations. Chest 1949; 15: 657-668.

4 Ikeda S. Atlas of Flexible Bronchofiberoscopy. Tokyo, IgakuShoin, 1974; pp. 58-71.

5 Jackson CL, Huber JF. Correlated applied anatomy of the bronchial tree and lungs with a system of nomenclature. Chest 1943; 9: 319-326.

6 Tschirren J, McLennan G, Palagyi K, et al. Matching and anatomical labeling of human airway tree. IEEE Trans Med Imaging 2005; 24: 1540-1547.

7 James AL, Wenzel S. Clinical relevance of airway remodelling in airway diseases. Eur Respir J 2007; 30: 134-155.

8 Dame Carroll JR, Chandra A, Jones AS, et al. Airway dimensions measured from micro-computed tomography and high-resolution computed tomography. Eur Respir J 2006; 28: 712-720.

9 Berger P, Perot V, Desbarats P, et al. Airway wall thickness in cigarette smokers: quantitative thin-section CT assessment. Radiology 2005; 235: 1055-1064.

10 Kalender WA, Rienmuller R, Seissler W, et al. Measurement of pulmonary parenchymal attenuation - use of spirometric gating with quantitative CT. Radiology 1990; 175: 265-268.

11 James AL, Pare PD, Hogg JC. Effects of lung volume, bronchoconstriction, and cigarette smoke on morphometric airway dimensions. J Appl Physiol 1988; 64: 913-919.

12 Hogg JC, Macklem PT, Thurlbeck WM. Site and nature of airway obstruction in chronic obstructive lung disease. $N$ Engl J Med 1968; 278: 1355-1360.

13 James AL, Pare PD, Hogg JC. The mechanics of airway narrowing in asthma. Am Rev Respir Dis 1989; 139: 242-246.

14 Yanai M, Sekizawa K, Ohrui T, et al. Site of airway obstruction in pulmonary disease: direct measurement of intrabronchial pressure. J Appl Physiol 1992; 72: 1016-1023.
15 Long FR, Williams RS, Castile RG. Structural airway abnormalities in infants and young children with cystic fibrosis. J Pediatr 2004; 144: 154-161.

16 Todo G, Herman PG. High-resolution computed tomography of the pig lung. Invest Radiol 1986; 21: 689-696.

17 King GG, Muller NL, Pare PD. Evaluation of airways in obstructive pulmonary disease using high-resolution computed tomography. Am J Respir Crit Care Med 1999; 159: 992-1004.

18 King GG, Muller NL, Whittall KP, et al. An analysis algorithm for measuring airway lumen and wall areas from high-resolution computed tomographic data. Am J Respir Crit Care Med 2000; 161: 574-580.

19 McNamara AE, Muller NL, Okazawa M, et al. Airway narrowing in excised canine lungs measured by high-resolution computed tomography. J Appl Physiol 1992; 73: 307-316.

20 Nakano Y, Muro S, Sakai H, et al. Computed tomographic measurements of airway dimensions and emphysema in smokers. Correlation with lung function. Am J Respir Crit Care Med 2000; 162: 1102-1108.

21 Nakano Y, Wong JC, de Jong PA, et al. The prediction of small airway dimensions using computed tomography. Am J Respir Crit Care Med 2005; 171: 142-146.

22 Palagyi K, Tschirren J, Hoffman EA, et al. Quantitative analysis of pulmonary airway tree structures. Comput Biol Med 2006; 36: 974-996.

23 Tschirren J, Hoffman EA, McLennan G, et al. Intrathoracic airway trees: segmentation and airway morphology analysis from low-dose CT scans. IEEE Trans Med Imaging 2005; 24: 15291539.

24 Bankier AA, Fleischmann D, Mallek R, et al. Bronchial wall thickness: appropriate window settings for thin-section CT and radiologic-anatomic correlation. Radiology 1996; 199: 831-836.

25 Niimi A, Matsumoto H, Amitani R, et al. Airway wall thickness in asthma assessed by computed tomography. Relation to clinical indices. Am J Respir Crit Care Med 2000; 162: 1518-1523.

26 Seneterre E, Paganin F, Bruel JM, et al. Measurement of the internal size of bronchi using high resolution computed tomography (HRCT). Eur Respir J 1994; 7: 596-600.

27 Webb WR, Gamsu G, Wall SD, et al. CT of a bronchial phantom. Factors affecting appearance and size measurements. Invest Radiol 1984; 19: 394-398.

28 Brown RH, Herold CJ, Hirshman CA, et al. In vivo measurements of airway reactivity using high-resolution computed tomography. Am Rev Respir Dis 1991; 144: 208-212.

29 McNittGray MF, Goldin JG, Johnson TD, et al. Development and testing of image-processing methods for the quantitative assessment of airway hyperresponsiveness from high-resolution CT images. J Comput Assist Tomogr 1997; 21: 939-947.

30 Amirav I, Kramer SS, Grunstein MM, et al. Assessment of methacholine-induced airway constriction by ultrafast highresolution computed tomography. J Appl Physiol 1993; 75: 22392250.

31 Montaudon M, Berger P, de Dietrich G, et al. Assessment of airways with three-dimensional quantitative thin-section CT: in vitro and in vivo validation. Radiology 2007; 242: 563-572.

32 Reinhardt JM, D'Souza ND, Hoffman EA. Accurate measurement of intrathoracic airways. IEEE Trans Med Imaging 1997; 16: 820-827.

33 Saba OI, Hoffman EA, Reinhardt JM. Maximizing quantitative accuracy of lung airway lumen and wall measures obtained from X-ray CT imaging. J Appl Physiol 2003; 95: 1063-1075.

34 de Jong PA, Muller NL, Pare PD, et al. Computed tomographic imaging of the airways: relationship to structure and function. Eur Respir J 2005; 26: 140-152.

35 Nakano Y, Muller NL, King GG, et al. Quantitative assessment of airway remodeling using high-resolution CT. Chest 2002; 122: 271S-275S. 
36 Venkatraman R, Raman R, Raman B, et al. Fully automated system for three-dimensional bronchial morphology analysis using volumetric multidetector computed tomography of the chest. J Digit Imaging 2006; 19: 132-139.

37 Bushberg JT, Seibert JA, Leidholdt EM Jr, et al., The Essential Physics of Medical Imaging. 2nd Edn. Philadelphia, Lippincott Williams and Wilkins, 2002; pp. 371-372.

38 Okazawa M, Muller N, McNamara AE, et al. Human airway narrowing measured using high resolution computed tomography. Am J Respir Crit Care Med 1996; 154: 1557-1562.

39 Wood SA, Zerhouni EA, Hoford JD, et al. Measurement of threedimensional lung tree structures by using computed tomography. J Appl Physiol 1995; 79: 1687-1697.

40 Tschirren J, Hoffman EA, McLennan G, et al. Segmentation and quantitative analysis of intrathoracic airway trees from computed tomography images. Proc Am Thorac Soc 2005; 2: 484-487.

41 Graham SM, McLennan G, Funk GF, et al. Preoperative assessment of obstruction with computed tomography image analysis. Am J Otolaryngol 2000; 21: 263-270.

42 Di Simone MP, Mattioli S, D'Ovidio F, et al. Three-dimensional CT imaging and virtual endoscopy for the placement of selfexpandable stents in oesophageal and tracheobronchial neoplastic stenoses. Eur J Cardiothorac Surg 2003; 23: 106-108.

43 Zwischenberger JB, Wittich GR, van Sonnenberg E, et al. Airway simulation to guide stent placement for tracheobronchial obstruction in lung cancer. Ann Thorac Surg 1997; 64: 1619-1625.

44 Hoppe H, Dinkel HP, Walder B, et al. Grading airway stenosis down to the segmental level using virtual bronchoscopy. Chest 2004; 125: 704-711.

45 Shitrit D, Valdsislav P, Grubstein A, et al. Accuracy of virtual bronchoscopy for grading tracheobronchial stenosis: correlation with pulmonary function test and fiberoptic bronchoscopy. Chest 2005; 128: 3545-3550.

46 Heyer CM, Nuesslein TG, Jung D, et al. Tracheobronchial anomalies and stenoses: detection with low-dose multidetector CT with virtual tracheobronchoscopy - comparison with flexible tracheobronchoscopy. Radiology 2007; 242: 542-549.

47 Hoppe H, Walder B, Sonnenschein M, et al. Multidetector CT virtual bronchoscopy to grade tracheobronchial stenosis. AJR Am J Roentgenol 2002; 178: 1195-1200.

48 Maniatis PN, Triantopoulou CC, Tsalafoutas IA, et al. Virtual bronchoscopy versus thin section computed-tomography in evaluation of moderate and low grade stenoses: receiver operating characteristic curve analysis. Acta Radiol 2006; 47: 48-57.

49 Burke AJ, Vining DJ, McGuirt WF, et al. Evaluation of airway obstruction using virtual endoscopy. Laryngoscope 2000; 110: 23-29.

50 Gilkeson RC, Ciancibello LM, Hejal RB, et al. Tracheobronchomalacia: dynamic airway evaluation with multidetector CT. AJR Am J Roentgenol 2001; 176: 205-210.

51 Zhang J, Hasegawa I, Feller-Kopman D, et al. 2003 AUR Memorial Award. Dynamic expiratory volumetric CT imaging of the central airways: comparison of standard-dose and lowdose techniques. Acad Radiol 2003; 10: 719-724.

52 Baroni RH, Feller-Kopman D, Nishino M, et al. Tracheobronchomalacia: comparison between end-expiratory and dynamic expiratory CT for evaluation of central airway collapse. Radiology 2005: 235; 635-641.

53 Carden KA, Boiselle PM, Waltz DA, et al. Tracheomalacia and tracheobronchomalacia in children and adults: an in-depth review. Chest 2005; 127: 984-1005.

54 Dumon JF. A dedicated tracheobronchial stent. Chest 1990; 97: 328-332.

55 Clarke DB. Palliative intubation of the trachea and main bronchi. J Thorac Cardiovasc Surg 1980; 80: 736-741.

56 Orlowski TM. Palliative intubation of the tracheobronchial tree. J Thorac Cardiovasc Surg 1987; 94: 343-348.
57 Ferretti GR, Kocier M, Calaque O, et al. Follow-up after stent insertion in the tracheobronchial tree: role of helical computed tomography in comparison with fiberoptic bronchoscopy. Eur Radiol 2003; 13: 1172-1178.

58 Wadsworth SJ, Juniper MC, Benson MK, et al. Fatal complication of an expandable metallic bronchial stent. Br J Radiol 1999; 72 : 706-708.

59 Dorffel WV, Fietze I, Hentschel D, et al. A new bronchoscopic method to measure airway size. Eur Respir J 1999; 14: 783-788.

60 McFawn PK, Forkert L, Fisher JT. A new method to perform quantitative measurement of bronchoscopic images. Eur Respir J 2001; 18: 817-826.

61 Doolin EJ, Strande LL. Calibration of endoscopic images. Ann Otol Rhinol Laryngol 1995; 104: 19-23.

62 Santos MC, Strande L, Doolin EJ. Airway measurement using morphometric analysis. Ann Otol Rhinol Laryngol 1995; 104: 835838.

63 Masters IB, Eastburn MM, Francis PW, et al. Quantification of the magnification and distortion effects of a pediatric flexible videobronchoscope. Respir Res 2005; 6: 16.

64 Forkert L, Watanabe $\mathrm{H}$, Sutherland $\mathrm{K}$, et al. Quantitative videobronchoscopy: a new technique to assess airway caliber. Am J Respir Crit Care Med 1996; 154: 1794-1803.

65 Riff EJ, Mitra S, Baker MC. Pediatric fiberoptic video bronchoscopy: the use of computer interfacing. Comput Biol Med 1993; 23: 345-347.

66 Helferty JP, Zhang C, McLennan G, et al. Videoendoscopic distortion correction and its application to virtual guidance of endoscopy. IEEE Trans Med Imaging 2001; 20: 605-617.

67 Mitchell HW, Sparrow MP. Video-imaging of lumen narrowing; muscle shortening and flow responsiveness in isolated bronchial segments of the pig. Eur Respir J 1994; 7: 1317-1325.

68 Czaja P, Soja J, Grzanka P, et al. Assessment of airway caliber in quantitative videobronchoscopy. Respiration 2007; 74: 432-438.

69 Masters IB, Eastburn MM, Wootton R, et al. A new method for objective identification and measurement of airway lumen in paediatric flexible videobronchoscopy. Thorax 2005; 60: 652-658

70 Rasband WS. ImageJ. Bethesda, US National Institutes of Health. http://rsb.info.nih.gov/ij/

71 Masters IB, Zimmerman PV, Chang AB. Longitudinal quantification of growth and changes in primary tracheobronchomalacia sites in children. Pediatr Pulmonol 2007; 42: 906-913.

72 Suter M, McLennan G, Reinhardt JM, et al. Macro-optical color assessment of the pulmonary airways with subsequent threedimensional multidetector-X-ray-computed-tomography assisted display. J Biomed Opt 2005; 10: 051703.

73 Suter M, Reinhardt JM, McLennan G. Integrated CT/bronchoscopy in the central airways: Preliminary results. Acad Radiol 2008; 15: 786-798.

74 Sheppard CJR, Shotton DM. Confocal Laser Scanning Microscopy. Oxford, Bios Scientific, 1997.

75 Flusberg BA, Cocker ED, Piyawattanametha W, et al. Fiber-optic fluorescence imaging. Nat Methods 2005; 2: 941-950.

76 Thiberville L, Moreno-Swirc S, Vercauteren $\mathrm{T}$, et al. In vivo imaging of the bronchial wall microstructure using fibered confocal fluorescence microscopy. Am J Respir Crit Care Med 2007; 175: 22-31.

77 Vercauteren T, Perchant A, Malandain G, et al. Robust mosaicing with correction of motion distortions and tissue deformations for in vivo fibered microscopy. Med Image Anal 2006; 10: 673-692.

78 Sampson DD, Hillman TR. Optical coherence tomography. In: Palumbo G, Pratesi R, eds. Lasers and Current Optical Techniques in Biology. Cambridge, Royal Society of Chemistry, 2004; pp. 481-571.

79 Fujimoto JG, Boppart SA, Tearney GJ, et al. High resolution in vivo intra-arterial imaging with optical coherence tomography. Heart 1999; 82: 128-133. 
80 Huang D, Swanson EA, Lin CP, et al. Optical coherence tomography. Science 1991; 254: 1178-1181.

81 Drexler W, Fujimoto JG. Optical coherence tomography in ophthalmology. J Biomed Opt 2007; 12: 041201.

82 Chen J, Lee L. Clinical applications and new developments of optical coherence tomography: an evidence-based review. Clin Exp Optom 2007; 90: 317-335.

83 Evans JA, Poneros JM, Bouma BE, et al. Optical coherence tomography to identify intramucosal carcinoma and high-grade dysplasia in Barrett's esophagus. Clin Gastroenterol Hepatol 2006; 4: $38-43$.

84 Bhindi R, Munir SM, Channon KM. Optical coherence tomography in the setting of an acute anterior myocardial infarction. Circulation 2007; 116: e366-e367.

85 Brezinski ME. Optical coherence tomography for identifying unstable coronary plaque. Int J Cardiol 2006; 107: 154-165.

86 Kubo T, Imanishi T, Takarada S, et al. Assessment of culprit lesion morphology in acute myocardial infarction: ability of optical coherence tomography compared with intravascular ultrasound and coronary angioscopy. J Am Coll Cardiol 2007; 50: 933-939.

87 Yun SH, Tearney GJ, Vakoc BJ, et al. Comprehensive volumetric optical microscopy in vivo. Nat Med 2006; 12: 1429-1433.

88 Han SG, El-Abbadi NH, Hanna N, et al. Evaluation of tracheal imaging by optical coherence tomography. Respiration 2005; 72: 537-541.

89 Hanna N, Saltzman D, Mukai D, et al. Two-dimensional and 3dimensional optical coherence tomographic imaging of the airway, lung, and pleura. J Thorac Cardiovasc Surg 2005; 129: 615-622.

90 Whiteman SC, Yang Y, van Pittius DG, et al. Optical coherence tomography: real-time imaging of bronchial airways microstructure and detection of inflammatory/neoplastic morphologic changes. Clin Cancer Res 2006; 12: 813-818.

91 Coxson HO, Quiney B, Sin DD, et al. Airway wall thickness assessed using computed tomography and optical coherence tomography. Am J Respir Crit Care Med 2008; 177: 1201-1206.

92 Armstrong JJ, Leigh MS, Walton ID, et al. In vivo size and shape measurement of the human upper airway using endoscopic longrange optical coherence tomography. Opt Express 2003;11: 1817-1826.

93 Leigh MS, Armstrong JJ, Paduch A, et al. Anatomical optical coherence tomography for long-term, portable, quantitative endoscopy. IEEE Trans Biomed Eng 2008; 55: 1438-1446.

94 Armstrong JJ, Leigh MS, Sampson DD, et al. Quantitative upper airway imaging with anatomic optical coherence tomography. Am J Respir Crit Care Med 2006; 173: 226-233.

95 Walsh JH, Leigh MS, Paduch A, et al. Effect of body posture on pharyngeal shape and size in adults with and without obstructive sleep apnoea. Sleep 2008; 31: 1543-1549.

96 Walsh JH, Leigh MS, Paduch A, et al. Evaluation of pharyngeal shape and size using anatomical optical coherence tomography in individuals with and without obstructive sleep apnoea. J Sleep Res 2008; 17: 230-238.

97 McLaughlin RA, Williamson JP, Phillips MJ, et al. Applying anatomical optical coherence tomography to quantitative 3D imaging of the lower airway. Opt Express 2008; 16: 17521-17529.

98 Bailey DL. Imaging the airways in 2006. J Aerosol Med 2006; 19: 1-7.

99 Yablonskiy DA, Sukstanskii AL, Leawoods JC, et al. Quantitative in vivo assessment of lung microstructure at the alveolar level with hyperpolarized He-3 diffusion MRI. Proc Natl Acad Sci USA 2002; 99: 3111-3116.

100 Tooker AC, Hong KS, McKinstry EL, et al. Distal airways in humans: dynamic hyperpolarized He-3 MR imaging - feasibility. Radiology 2003; 227: 575-579.

101 Lewis TA, Tzeng YS, McKinstry EL, et al. Quantification of airway diameters and 3D airway tree rendering from dynamic hyperpolarized He-3 magnetic resonance imaging. Magn Reson Med 2005; 53: 474-478.
102 Lakhal K, Delplace X, Cottier JP, et al. The feasibility of ultrasound to assess subglottic diameter. Anesth Analg 2007; 104: 611-614.

103 Silverstein FE, Martin RW, Kimmey MB, et al. Experimental evaluation of an endoscopic ultrasound probe - in vitro and in vivo canine studies. Gastroenterology 1989; 96: 1058-1062.

104 Shaw TJ, Wakely SL, Peebles CR, et al. Endobronchial ultrasound to assess airway wall thickening: validation in vitro and in vivo. Eur Respir J 2004; 23: 813-817.

105 de Jong PA, Ottink MD, Robben SGF, et al. Pulmonary disease assessment in cystic fibrosis: comparison of CT scoring systems and value of bronchial and arterial dimension measurements Radiology 2004; 231: 434-439.

106 Helbich TH, Heinz-Peer G, Fleischmann D, et al. Evolution of CT findings in patients with cystic fibrosis. Am J Roentgenol 1999; 173: 81-88.

107 de Jong PA, Nakano Y, Lequin MH, et al. Progressive damage on high resolution computed tomography despite stable lung function in cystic fibrosis. Eur Respir J 2004; 23: 93-97.

108 Brody AS, Klein JS, Molina PL, et al. High-resolution computed tomography in young patients with cystic fibrosis: distribution of abnormalities and correlation with pulmonary function tests. J Pediatr 2004; 145: 32-38.

109 de Gonzalez AB, Kim KP, Samet JM. Radiation-induced cancer risk from annual computed tomography for patients with cystic fibrosis. Am J Respir Crit Care Med 2007; 176: 970-973.

110 de Jong PA, Mayo JR, Golmohammadi K, et al. Estimation of cancer mortality associated with repetitive computed tomography scanning. Am J Respir Crit Care Med 2006; 173: 199-203.

111 de Jong PA, Nakano Y, Lequin MH, et al. Dose reduction for CT in children with cystic fibrosis: is it feasible to reduce the number of images per scan? Pediatr Radiol 2006; 36: 50-53.

112 Jimenez S, Jimenez JR, Crespo $M$, et al. Computed tomography in children with cystic fibrosis: a new way to reduce radiation dose. Arch Dis Child 2006; 91: 388-390.

113 Tiddens $\mathrm{H}$, de Jong PA. Update on the application of chest computed tomography scanning to cystic fibrosis. Curr Opin Pulm Med 2006; 12: 433-439.

114 Fain SB, Gonzalez-Fernandez G, Peterson ET, et al. Evaluation of structure-function relationships in asthma using multidetector CT and hyperpolarized He-3 MRI. Acad Radiol 2008; 15: 753-762.

115 Gono H, Fujimoto K, Kawakami S, et al. Evaluation of airway wall thickness and air trapping by HRCT in asymptomatic asthma. Eur Respir J 2003; 22: 965-971.

116 Ueda T, Niimi A, Matsumoto H, et al. Role of small airways in asthma: investigation using high-resolution computed tomography. J Allergy Clin Immunol 2006; 118: 1019-1025.

117 Hogg JC, Chu F, Utokaparch S, et al. The nature of small-airway obstruction in chronic obstructive pulmonary disease. $N$ Engl $J$ Med 2004; 350: 2645-2653.

118 Jeffery PK. Structural and inflammatory changes in COPD: a comparison with asthma. Thorax 1998; 53: 129-136.

119 Kitaguchi Y, Fujimoto K, Kubo K, et al. Characteristics of COPD phenotypes classified according to the findings of HRCT. Respir Med 2006; 100: 1742-1752.

120 Hoffman EA, Simon BA, McLennan G. State of the art. A structural and functional assessment of the lung via multidetector-row computed tomography: phenotyping chronic obstructive pulmonary disease. Proc Am Thorac Soc 2006; 3: 519-532.

121 Orlandi I, Moroni C, Camiciottoli G, et al. Chronic obstructive pulmonary disease: thin-section CT measurement of airway wall thickness and lung attenuation. Radiology 2005; 234: 604-610.

122 Arakawa A, Yamashita Y, Nakayama Y, et al. Assessment of lung volumes in pulmonary emphysema using multidetector helical CT: comparison with pulmonary function tests. Comput Med Imaging Graph 2001; 25: 399-404. 
123 Kauczor HU, Hast J, Heussel CP, et al. CT attenuation of paired HRCT scans obtained at full inspiratory/expiratory position: comparison with pulmonary function tests. Eur Radiol 2002; 12 $2757-2763$.
124 Zaporozhan J, Ley S, Eberhardt R, et al. Paired inspiratory/ expiratory volumetric thin-slice CT scan for emphysema analysis - comparison of different quantitative evaluations and pulmonary function test. Chest 2005; 128: 3212-3220. 